\title{
The functional role of alternation advantage in the sequence effect of symbolic cueing with nonpredictive arrow cues
}

\author{
Qian Qian • Miao Song • Keizo Shinomori • Feng Wang
}

Published online: 21 June 2012

(C) Psychonomic Society, Inc. 2012

\begin{abstract}
Previous studies have shown that attention orienting is influenced by the orienting processes of previous trials in a spatial-cueing paradigm. This sequence effect is due to the fact that performance is facilitated when cue validity (valid or invalid) repeats between trials. In this study, we investigated the influences of cue direction and target location on the sequence effect of symbolic cueing with a nonpredictive central arrow cue. The cue direction was manipulated to always point in a certain direction in one condition, and to always point in the opposite direction in a second condition. The results showed that sequence effects were enhanced by the alternation of cue direction and target location and were impaired by the repetition of cue direction and target location. The same result pattern was found when the cue direction was chosen randomly in the third condition. The results suggested that both the repetition advantage effect of cue validity and the alternation advantage effect of cue direction and target location are involved in the sequence effect within the symboliccueing paradigm.
\end{abstract}

\footnotetext{
Q. Qian $(\bowtie) \cdot$ F. Wang

Yunnan Key Laboratory of Computer Technology Applications, Kunming University of Science and Technology,

Cheng Gong Da Xue Cheng,

Kunming, Yunnan 650500, China

e-mail: qianqian1025@gmail.com

Q. Qian $\cdot$ M. Song $\cdot$ K. Shinomori

Department of Information Systems Engineering, Kochi

University of Technology,

Tosayamada-town,

Kami-city, Kochi 782-8502, Japan

M. Song

Information Engineering College, Shanghai Maritime University,

Shanghai 200135, China
}

Keywords Sequence effect · Arrow cueing · Attention orienting $\cdot$ Cueing effect

Although some early studies suggested that very little visual information is explicitly retained across views (see, e.g., Ballard, Hayhoe, \& Pelz, 1995; Grimes, 1996), a number of more recent studies have consistently shown that attention allocation is heavily influenced by the most recently viewed stimuli that were important for behavior (e.g., Chun \& Nakayama, 2000; Wolfe, Butcher, Lee, \& Hyle, 2003). For instance, Maljkovic and Nakayama (1994) found that in searching for a color singleton target, when target and nontarget colors are switched unpredictably from trial to trial, response is faster in a trial on which the target color is the same as in the preceding trial than in a trial on which the target color is different, a phenomenon that they called priming of pop-out ( $\mathrm{PoP})$. Besides color, this sequence effect on visual search performance has also been observed in investigations of other properties, such as orientation (Hillstrom, 2000), shape (Lamy, Carmel, Egeth, \& Leber, 2006), location (Maljkovic \& Nakayama, 1996), and even emotional expression (Lamy, Amunts, \& Bar-Haim, 2008). PoP is generally believed to be afforded by implicit visual memory mechanisms and without voluntary intervention (Kristjánsson, 2006). Another good demonstration of sequence effects between trials is negative priming (e.g., Neill \& Valdes, 1992; Tipper, 2001), which refers to the phenomenon wherein a target stimulus is more slowly responded to on a current trial when the same stimulus was ignored on a previous trial. Negative priming has mainly been explained as selective inhibition or episodic retrieval (Egner \& Hirsch, 2005). All of these studies have shown that some crucial information from previous views could be used to guide attention allocation shortly afterward. 
Sequence effects between trials have also been found in the central-cueing paradigm, in which observers respond to a left or right target following a valid or an invalid central symbolic cue that indicated the possible target locations. By using a predictive central arrow cue (i.e., as $80 \%$ ratio of valid trials among all trials), Jongen and Smulders (2007) reported that the cueing effect (i.e., mean RT of invalid trials - mean RT of valid trials) was larger after a valid trial than after an invalid trial. They explained this sequence effect as momentary strategic adjustments in which participants adapt their utilization of the cue depending on whether it had correctly or wrongly directed their attention on the previous trial. Specifically, a valid trial enhances the expectation for repetitions, so that it is beneficial to direct attention to the cued location, whereas an invalid trial weakens this expectation, or even promotes orienting to the uncued location. Similar sequence effect patterns have also been found in the studies of Gómez and his colleagues (Arjona \& Gómez, 2011; Gómez \& Flores, 2011; Gómez, Flores, Digiacomo, \& Vázquez-Marrufo, 2009). Compatible with the explanation of Jongen and Smulders, these studies attributed the sequence effect to the continuous updating of the predictive value that participants assign to the spatial cue. All of these studies explained the sequence effect as a kind of top-down control exerted by participants, since the cues within these studies successfully predicted the target locations in most trials; however, the sequence effect observed in these studies can also be explained well by automatic memory processes (Logan, 1988) in which information from previous trials is automatically retrieved from memory to facilitate performance on current trials. For example, when the previous trial type (valid or invalid) is consistent with the current trial type, performance will be facilitated, whereas when the previous and current trial types differ, performance is slowed due to the conflict between the two trial types. This automatic-retrieval hypothesis is in line with the view from exogenous-cueing studies that have used peripheral cues (e.g., Dodd \& Pratt, 2007) and is further supported by the results of our previous study (Qian, Shinomori, \& Song, 2012), in which the sequence effect was still found when the arrow cues did not predict the target location and participants were explicitly asked to ignore the arrow cues.

One important issue in the investigation of sequence effects in a cueing paradigm is the influence of the cue direction and target location. According to the automaticretrieval hypothesis, the sequence effect is interpreted as the repetition advantage effect of cue validity between trials. However, when we take the cue direction and target location into account, two other possible repetition conditions exist. For example, following a valid trial in which both the cue direction and target location were to the left, the cue direction and target location of the next valid trial could both be left again or could both be right. In the former condition, cue validity, cue direction, and target location all repeat at the same time, but in the latter condition, only the cue validity of the trial repeats; the cue direction and target location both switch. The same circumstances can be found for trial pairs with repeated invalid cues. Whether or not the sequence effect of cueing paradigm is influenced by the repetition or switch of cue direction and target location has not been well investigated.

Jongen and Smulders (2007) did not directly investigate the influence of cue direction and target location on the sequence effect. Though they conducted an ANOVA on RTs and found a significant four-way interaction between previous cue direction, previous target location, current cue direction, and current target location-which indicated sequence effects - the potential influences of cue direction and target location on the sequence effects could not be seen in this analysis. In our previous study (Qian et al., 2012), we found a tendency for the sequence effect to be stronger when the cue direction between trials switched, as compared with when it repeated. However, this tendency failed to reach significance $(F=2.014, p=.17)$. This failure to show a significant influence may be attributed to two factors. First, the number of participants was relatively low (only 16). Second, the time interval between the arrow cues and targets (stimulus onset asynchrony [SOA]) was either 100 or $700 \mathrm{~ms}$, and the sequence effect in that study was not significant when the SOA of the previous trial was $100 \mathrm{~ms}$. This means that the sequence effect was not shown during half of the trials in that study, and this may have hindered the investigation into the influence of cue direction and target location. Therefore, we were motivated to carry out a systematic investigation of the influence of cue direction and target location on the sequence effect of arrow cueing. Such an investigation is important because it may reveal the detailed mechanisms underlying sequence effects and may provide better understanding of the central-cueing paradigm for future research.

Traditionally, a centrally presented arrow cue has been thought to orient attention only when it explicitly predicted the target location (Jonides, 1981; Posner, Snyder, \& Davidson, 1980). However, many recent studies have proved that a nonpredictive symbolic cue, such as an arrow, can induce cueing effects automatically (e.g., Hommel, Pratt, Colzato, \& Godijn, 2001; Pratt \& Hommel, 2003; Ristic, Friesen, \& Kingstone, 2002; Tipples, 2002). Although the cueing effects of a nonpredictive symbolic cue and those of a peripheral cue are very similar, many subtle differences are also to be found, such as the prolonged time course and the absence of an inhibition-of-return effect for symbolic cueing. As a result, some researchers have suggested that the attention orienting induced by centrally presented symbolic cues is controlled by a so-called symbolic-orienting mechanism, which is different from the exogenous-orienting and endogenous-orienting mechanisms (Ristic \& Kingstone, 
2012). As for the sequence effects within a cueing paradigm, similar effect patterns have been found when peripheral cues and arrow cues are used for cueing attention. In this study, we used a nonpredictive arrow as the central cue for the following reasons. First, our previous study proved that a nonpredictive arrow cue is capable of inducing sequence effects. Second, by using a nonpredictive cue, we could reduce the influence of participants' voluntary control and focus on the implicit mechanisms that we wanted to measure. Third, we intended to investigate the unknown mechanisms underlying symbolic orienting. As mentioned above, a symbolic cue, such as a nonpredictive arrow, may orient attention in a different way from that of a peripheral cue, even though both cues are capable of inducing cueing effects and sequence effects reflexively. In a peripheral-cueing study, Dodd and Pratt (2007) did not find any influence of cue direction and target location on sequence effects. We wanted to investigate whether the same result could be obtained for symbolic cueing.

The aim of the present study was to investigate the potential influence of repetitions or switches of cue direction and target location on sequence effects in arrow cueing. Three conditions with different manipulations of cue directions were tested. In the first condition, the arrows always cued the same location within a block (either left or right), while the target locations remained random. Therefore, following a valid or invalid trial, a trial with repeated cue validity would always have the same cue direction and target location as the previous trial. In the second condition, the arrows always cued a different location than that of the previous arrow (i.e., the cue sequence was "... left, right, left, right ..."), while the target locations remained random. Therefore, when cue validity repeated on consecutive trials, both the cue direction and the target location alternated. In the third condition, the previous two conditions were randomly combined to form a common cueing procedure, in which both cue directions and target locations remained random.

Notice that in the first and second conditions, the cue direction on each trial was manipulated depending on the cue direction of previous trials. Therefore, cue direction uncertainty was lost. This disappearance of cue direction uncertainty should have no significant influence on the sequence effect, according to the automatic-retrieval hypothesis. However, if uncertainty about the cue direction is an important requirement for sequence effects, there should be no sequence effect in both of these conditions. Regarding the influence on repetition/switch effects of cue direction and target location, there are several possibilities. First, if the sequence effect depends only on the repetition advantage effect of cue validity, sequence effects of similar magnitudes should be observed for all three conditions. Second, if the repetition of the cue direction and the target location can facilitate performance just like the repetition of cue validity, the first condition, with repeated cue directions, should induce stronger sequence effects than the other two conditions, because cue validity, cue direction, and target location all repeated at the same time. Third, many previous studies have reported that RTs were slower when the same target location or response was repeated than when it was switched (e.g., Fecteau, Au, Armstrong, \& Munoz, 2004; Maylor \& Hockey, 1987); that is, alternation of target location facilitates observers' performance, and repetition of target location slows observers' performance. If this alternation advantage effect of target location occurs in a cueing paradigm, the second condition, with alternated cue directions, should induce a stronger sequence effect than the other two conditions, because performance would be facilitated by both the repetition of cue validity and the accompanying alternation of cue direction and target location. In addition, under the first condition, with repeated cue direction, performance would be facilitated by the repetition of cue validity but slowed by the accompanying repetitions of cue direction and target location, resulting in an impaired sequence effect as compared with the other two conditions.

\section{Method}

\section{Participants}

A total of 20 students (with a mean age of 27 years, range 22 to 32 years; six females, 14 males) from Kochi University of Technology consented to participate in this experiment. All participants were right-handed and reported normal or corrected-to-normal vision. All were naive as to the purpose of the experiment.

\section{Apparatus}

The stimuli were presented on an LCD display operating at a $60-\mathrm{Hz}$ frame rate and controlled by a Dell Pentium computer. The participants were seated approximately $60 \mathrm{~cm}$ away from the screen.

\section{Stimuli}

A cross, subtending $1.5^{\circ}$, was placed at the center of the screen as a fixation point and remained on screen during the whole experiment. The cue was a pair of arrows pointing to either the left $(<<)$ or the right $(>>)$ that were positioned around the central cross and were $1.5^{\circ}$ in height and $5^{\circ}$ in width. The target stimulus was a capital letter " $T$ " measuring $1^{\circ}$ wide and $1^{\circ}$ high, which was presented $15^{\circ}$ from the fixation point on the left or the right side. 


\section{Design}

The cue-target SOA was $500 \mathrm{~ms}$. Three sessions were presented, with different manipulations of cue direction. Cue directions remained the same within blocks (but switched between blocks) in one session; they changed on every trial in another session; and they remained random in the third type of session. The sessions were randomly ordered for each participant. The numbers of blocks in each of the session types were two, two, and four, respectively. Each block included 112 trials in total, 16 of which were catch trials in which the target did not appear. The participants were instructed not to respond if the target did not appear. Including 20 training trials at the beginning of the experiment, this led to a total of 916 trials for each participant. The RTs of the first trial on each block and of trials following a catch trial were excluded from the analysis.

\section{Procedure}

The participants were instructed to keep fixating the center of the screen. First, a fixation display appeared at the center of the screen for $2,000 \mathrm{~ms}$, and then the cue stimulus appeared. After the cue-target SOA, a target letter "T" appeared to the left or the right either until participants had responded or until 1,500 ms had elapsed. The cue stimulus still remained on the screen after the appearance of the target. Participants were instructed to respond when the target appeared by pressing the space bar with the index finger of their right hand as quickly and accurately as possible. The participants were explicitly told about the manipulation of cue direction in each session. They were also informed that the central stimuli did not predict the location at which the target would appear and that they should try to ignore the central cues.

\section{Results}

The participants missed an average of about $0.1 \%$ of the targets and made false alarm errors on approximately $1.2 \%$ of the catch trials. Anticipations (RTs of less than $100 \mathrm{~ms}$ ) and outliers (RTs over 1,000 ms) were classified as errors and were excluded from the analysis. As a result, about $0.5 \%$ of all trials were removed. The error rates did not vary systematically, and no signs of any speed-accuracy trade-off were observed.

The mean RTs under different conditions can be seen in Table 1. A three-way ANOVA with Previous Cue Validity (pre-valid or pre-invalid), Cue Validity (valid or invalid), and Cue Direction Manipulation (always repeat, always switch, or random) as within-participants factors was conducted on the RTs. We found a main effect of cue validity, $F$
$(1,19)=16.998, p<.001$, indicating cueing effects; that is, RTs were shorter in valid than in invalid trials. There was a significant interaction between previous cue validity and cue validity, $F(1,19)=6.012, p<.024$, demonstrating that the cueing effect was stronger after a valid trial than after an invalid trial - that is, a typical sequence effect, as reported in previous studies. Importantly, the three-way interaction of Previous Cue Validity $\times$ Cue Validity $\times$ Cue Direction Manipulation was significant, $F(2,38)=7.844, p<.001$, indicating that the sequence effect was influenced by the manipulation condition of the cue directions. The magnitude of the cueing effects under different conditions can be seen from Fig. 1: The magnitudes of the sequence effects (i.e., cueing effects of pre-valid trials - cueing effects of preinvalid trials) for the cue manipulation conditions were $5 \mathrm{~ms}$ (always repeat), $29 \mathrm{~ms}$ (always switch), and $9 \mathrm{~ms}$ (random). Paired-samples $t$ tests proved, firstly, that the sequence effect when cue directions always switched was stronger than the sequence effects when cue direction always repeated or remained random $(p<.002$ and $p=.055$, respectively), and secondly, that the sequence effect when cue directions remained random was stronger than the sequence effect when cue direction always repeated $(p<.033)$. The results showed that sequence effects were impaired when the cue direction always repeated between trials and were enhanced when the cue direction always switched between trials.

When the cue direction was chosen randomly, the cue direction between trials could be either repeated or switched. This gave us an opportunity to investigate the influences of cue direction and target location without the loss of cue direction uncertainty. A three-way ANOVA with Previous Cue Validity (pre-valid or pre-invalid), Cue Validity (valid or invalid), and Cue Condition (cue direction the same as or different from the previous trial) as within-participants factors was conducted on RTs from the third condition, with random cue directions. The main effects of previous cue validity and cue validity were significant, $F(1,19)=7.962$, $p<.011$, and $F(1,19)=12.475, p<.002$, respectively, indicating that RTs were shorter on pre-invalid than on prevalid trials, and were shorter on valid than on invalid trials. Importantly, the Previous Cue Validity $\times$ Cue Validity $\times$ Cue Condition interaction was also significant, $F(1,19)=5.722$, $p<.027$, replicating the previous observation that the sequence effect was significant when the cue direction switched, but not when the cue direction repeated. The magnitudes of the cueing effects under different conditions can be seen in Fig. 2, and the magnitudes of the sequence effects for the cue conditions were $-6 \mathrm{~ms}$ (repeat) versus $16 \mathrm{~ms}$ (switch).

In order to investigate whether the RTs and cueing effects were different between the different cue direction manipulation conditions, paired-samples $t$ tests were conducted on 
Table 1 Mean RTs under different conditions in our experiment

\begin{tabular}{|c|c|c|c|c|c|c|c|c|}
\hline \multirow{3}{*}{$\begin{array}{l}\text { Cue Direction } \\
\text { Conditions }\end{array}$} & \multicolumn{4}{|c|}{ Pre-Valid } & \multicolumn{4}{|c|}{ Pre-Invalid } \\
\hline & \multicolumn{2}{|c|}{ Valid } & \multicolumn{2}{|c|}{ Invalid } & \multicolumn{2}{|c|}{ Valid } & \multicolumn{2}{|c|}{ Invalid } \\
\hline & $M$ & $S D$ & $M$ & $S D$ & $M$ & $S D$ & $M$ & $S D$ \\
\hline Always repeat & 358 & 51 & 366 & 60 & 357 & 52 & 370 & 60 \\
\hline Always switch & 348 & 61 & 375 & 72 & 361 & 73 & 358 & 63 \\
\hline Random & 346 & 65 & 357 & 70 & 346 & 65 & 349 & 64 \\
\hline \multicolumn{9}{|c|}{ Random divided } \\
\hline Repeat & 350 & 65 & 355 & 68 & 342 & 63 & 354 & 71 \\
\hline Switch & 343 & 64 & 357 & 71 & 346 & 65 & 344 & 59 \\
\hline
\end{tabular}

The upper part shows the RTs of three cue direction manipulation conditions: always repeat, always switch, and random. The lower part shows the RTs of two subconditions when cue direction was chosen randomly: The cue direction either repeated or switched between trials.

the RTs and the cueing effects of two manipulation conditions: always repeat and always switch. The results showed that the overall RTs of participants did not significantly differ between the repeating and switching cue conditions, $t(19)=0.226, p>.824$ (the mean RTs of the two conditions were 363 and $361 \mathrm{~ms}$, respectively). The results also showed that the magnitudes of cueing effects did not significantly differ between the two conditions, $t(19)=0.483, p>.635$ (the mean cueing effects of the two conditions were 10 and $12 \mathrm{~ms}$ for the repeating and switching conditions, respectively).

\section{Discussion}

In the present study, we investigated whether the repetition or switch of cue direction and target location could influence the sequence effect in an arrow-cueing paradigm. The results showed that sequence effects were impaired when the cue direction always repeated between trials but were enhanced when the cue direction always switched between trials. Similar results were also found in the common cueing procedure in the third condition, in which the cue direction

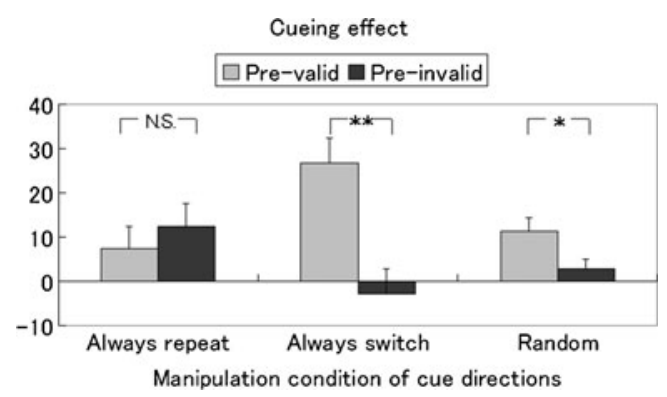

Fig. 1 Magnitudes of cueing effects $\left(\mathrm{RT}_{\text {uncued }}-\mathrm{RT}_{\text {cued }}\right)$ under different previous cue validity and cue manipulation conditions. The error bars denote standard errors of the means. ${ }^{*} p<.05$. ${ }^{* *} p<.01$ on each trial was chosen randomly. These results suggest that the sequence effect in the central-cueing paradigm cannot be solely attributed to the repetition advantage for cue validity, but is also influenced by the repetition/switch effect of cue direction and target location.

In the present experiment, the cue direction was manipulated between blocks, and one may argue that the results may have been influenced by such an experimental design. For example, in the block in which the cue always pointed to the same location, it is possible that participants would become less sensitive to the cue or would tend to ignore it altogether. The reduction of participants' attention may have reduced the likelihood of previous trial information being used in addition to how it was used. Therefore, the impaired sequence effect in the constant-repeating condition could be explained by the lack of attention to the cue. However, such an explanation is unlikely for the following reasons. First, the present results show that the overall RTs of participants did not significantly differ between the repeating and switching cue conditions. If participants' attention was modulated by the cue conditions, we should have observed different overall RTs between the two cue conditions, but this was not the case. Second, if participants tended to ignore the cue when the cue always pointed to the same location, smaller cueing effects should have been observed in the repeating cue condition than in the switching cue condition. However, the present results show that the magnitudes of the cueing effects, independent of previous cue validity, did not significantly differ between the repeating and switching cue conditions. Third, similar results were also found in the cueing procedure with random cue directions. These observations rule out the possible influence of block type and provide a strong support for our conclusion.

According to the automatic-retrieval hypothesis, information from previous trials is automatically retrieved from memory to facilitate performance on current trials. However, the present results suggest that the automaticretrieval hypothesis is not adequate to interpret all of the phenomena related to sequence effects in the cueing

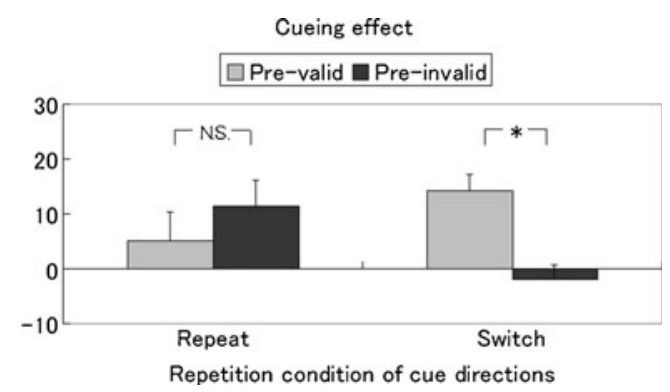

Fig. 2 Magnitudes of cueing effects under different repetition conditions of cue direction when cue directions remained random across trials. The asterisk marks a difference statistically significant at the $p<$ .001 level. The error bars denote standard errors of the means 
paradigm. In fact, the participants' performance was slowed, rather than facilitated, by the repetition of cue direction and target location. As a result, the sequence effects were influenced by this alternation advantage effect of cue direction and target location. Alternation advantage effects have been reported in many studies using both detection and choice RT tasks (e.g., Fecteau et al., 2004; Maylor \& Hockey, 1987). There are two explanations for the alternation advantage effect: guessing strategies of participants or inhibition mechanisms. According to the guessing-strategy explanation, participants expect that a target appearing at a particular location is more likely to be followed by a target appearing at the opposite location, even though the probabilities of either location are equal. In the present study, the participants already knew the cue direction of the next trial when the cue directions always repeated or switched between trials, so they did not need to guess the next cue direction. Therefore, if the alternation advantage effect is a result of guessing strategies, the present influence of the alternation advantage on the sequence effect can solely be attributed to the repetition/switch effect of target location, rather than to the repetition/switch effect of cue direction. On the other hand, if the alternation advantage effect is a result of inhibition mechanisms, then we cannot discriminate the repetition/switch effect of cue direction from that of target location. This is because when a certain cue validity status repeated between trials, the repetition (or switch) of cue direction always accompanied the repetition (or switch) of target location.

One thing that needs to be pointed out is that in an exogenous cueing paradigm, Dodd and Pratt (2007) failed to show a significant influence of target location on the sequence effect of peripheral cueing. The possible reasons for this are as follows. First, one critical difference between this study and peripheral-cueing studies is that the arrow cues were perceptually different but spatially similar, whereas peripheral cues are perceptually identical but their spatial locations differ. Shifts of attention by central cues tend to be slower than shifts to peripheral cues, and many studies have concluded that different processing mechanisms are involved between arrow cueing and peripheral cueing (e.g., Cheal \& Lyon, 1991; Jonides, 1981; Müller \& Rabbitt, 1989; Ristic \& Kingstone, 2012). Second, in the peripheral-cueing study of Dodd and Pratt, they examined inhibition-of-return effects rather than cueing effects. Commonly, we do not expect to see inhibition of return in arrow cueing, so it is likely that the mechanisms measured in the present study are different from those measured in Dodd and Pratt's study. Therefore, it is not surprising to find that the effect pattern observed in the present study only appeared for central arrow cues. In line with the present findings, in our previous study (Qian et al., 2012) we found that the sequence effect of arrow cueing was modulated by the cue-target SOA of the previous trial, which was different from the findings of peripheral-cueing tasks (Mordkoff, Halterman, \& Chen, 2008). The present study may have found new evidence of the difference between symbolic and exogenous cueing.

Until now, sequence effects in arrow cueing have been found when both predictive and nonpredictive arrow cues were tested. When using a predictive arrow cue, researchers can not discriminate automatic processes from the voluntary control processes of participants. Maybe this is why many previous studies attributed sequence effects to strategic changes or to participants' assignment of credibility to the cues, depending on the outcomes of previous trials. By using nonpredictive arrow cues, our previous and present studies have proved that sequence effects from arrow cueing can take effect without voluntary intervention. This result is in line with the findings of other studies that have focused on trial-by-trial effects in other paradigms, such as priming of pop-out (e.g., Kristjánsson, 2006; Maljkovic \& Nakayama, 2000) and negative priming (e.g., Egner \& Hirsch, 2005; Neill \& Valdes, 1992) in a visual-search paradigm. By the way, although the fact that sequence effects can be induced by nonpredictive symbolic cues suggests that sequence effects do not stem from the voluntary control of participants, we still can not rule out the possibility that some implicit top-down processes are involved in the sequence effect. Indeed, Gómez and his colleagues conducted a series of human electrophysiological studies (e.g., Gómez et al., 2009) to investigate the trial-bytrial changes of ERPs in Posner's central-cueing paradigm. They found that the contingent negative variation (an ERP component related to attention and preparation) is higher in pre-valid than in pre-invalid sequences, indicating that more attentional resources are deployed in pre-valid than in preinvalid trials. Therefore, there remains the question of whether top-down processes are influencing sequence effects. One possible research method would be to investigate whether the magnitude of sequence effects can be modulated by the voluntary control of participants or by experimental contexts. In our laboratory, we are preparing a new study to investigate the influence of the reliability of arrow cues on sequence effects. The sequence effects from nonpredictive arrow cues will be compared directly with the sequence effects from predictive or counterpredictive arrow cues. The results will help to reveal the origin of sequence effects and to provide a better understanding of human spatial-attention mechanisms.

During the past three decades, the spatial-cueing paradigm has been widely used in the study of attention orienting. However, very few studies have tried to investigate trial-by-trial effects in the cueing paradigm. In several previous studies (e.g., Dodd \& Pratt, 2007; Qian et al., 2012), researchers have attributed sequence effects to the 
repetition/switch effect of cue validity; however, the detailed mechanisms underlying the sequence effect are still unclear. By using a nonpredictive arrow cue, we sought to extend previous findings in the present study by demonstrating that the repetition/switch effect of cue direction and target location is also involved in the sequential processes of arrow cueing. The results may have revealed the potential mechanisms involved in the sequence effect in the symboliccueing paradigm, and they could be used to guide future cueing studies.

Author note The authors thank Michael Dodd, Carlos Gómez, and one anonymous reviewer for many helpful comments on a previous version of the manuscript. This research is supported by grants from JSPS KAKENHI (20300081 and 24300085), NSFC (61063027), and Applied Basic Research Foundation of Yunnan Province (KKSY201203026).

\section{References}

Arjona, A., \& Gómez, C. M. (2011). Trial-by-trial changes in a priori informational value of external cues and subjective expectancies in human auditory attention. PloS ONE, 6, e21033. doi:10.1371/ journal.pone.0021033

Ballard, D., Hayhoe, M., \& Pelz, J. (1995). Memory representation in natural tasks. Journal of Cognitive Neuroscience, 7, 66-80.

Cheal, M., \& Lyon, D. R. (1991). Central and peripheral precuing of forced-choice discrimination. Quarterly Journal of Experimental Psychology, 43A, 859-880. doi:10.1080/14640749108400960

Chun, M., \& Nakayama, K. (2000). On the functional role of implicit visual memory for the adaptive deployment of attention across scenes. Visual Cognition, 7, 65-81.

Dodd, M., \& Pratt, J. (2007). The effect of previous trial type on inhibition of return. Psychological Research, 71, 411-417. doi:10.1007/s00426-005-0028-0

Egner, T., \& Hirsch, J. (2005). Where memory meets attention: Neural substrates of negative priming. Journal of Cognitive Neuroscience, 17, 1774-1784.

Fecteau, J. H., Au, C., Armstrong, I. T., \& Munoz, D. P. (2004). Sensory biases produce alternation advantage found in sequential saccadic eye movement tasks. Experimental Brain Research, 159, 84-91.

Gómez, C. M., \& Flores, A. (2011). A neurophysiological evaluation of a cognitive cycle in humans. Neuroscience and Biobehavioral Reviews, 35, 452-461.

Gómez, C. M., Flores, A., Digiacomo, M. R., \& Vázquez-Marrufo, M. (2009). Sequential P3 effects in a Posner's spatial cueing paradigm: Trial-by-trial learning of the predictive value of the cue. Acta Neurobiologiae Experimentalis, 69, 155-167.

Grimes, J. (1996). On the failure to detect changes in scenes across saccades. In K. Akins (Ed.), Vancouver studies in cognitive science: Vol. 5. Perception (pp. 89-110). New York, NY: Oxford University Press.

Hillstrom, A. P. (2000). Repetition effects in visual search. Perception \& Psychophysics, 62, 800-817. doi:10.3758/ BF03206924

Hommel, B., Pratt, J., Colzato, L., \& Godijn, R. (2001). Symbolic control of visual attention. Psychological Science, 12, 360365 .

Jongen, E., \& Smulders, F. (2007). Sequence effects in a spatial cueing task: Endogenous orienting is sensitive to orienting in the preceding trial. Psychological Research, 71, 516523.

Jonides, J. (1981). Voluntary versus automatic control over the mind's eye's movement. In J. Long \& A. Baddeley (Eds.), Attention and performance $I X$ (pp. 187-203). Hillsdale, NJ: Erlbaum.

Kristjánsson, Á. (2006). Rapid learning in attention shifts: A review. Visual Cognition, 13, 324-362.

Lamy, D., Amunts, L., \& Bar-Haim, Y. (2008). Emotional priming of pop-out in visual search. Emotion, 8(2), 151-161.

Lamy, D., Carmel, T., Egeth, H. E., \& Leber, A. B. (2006). Effects of search mode and intertrial priming on singleton search. Perception \& Psychophysics, 68, 919-932. doi:10.3758/BF03193355

Logan, G. (1988). Toward an instance theory of automatization. Psychological Review, 95, 492-527. doi:10.1037/0033-295X.95.4.492

Maljkovic, V., \& Nakayama, K. (1994). Priming of pop-out: I. Role of features. Memory \& Cognition, 22, 657-672. doi:10.3758/ BF03209251

Maljkovic, V., \& Nakayama, K. (1996). Priming of pop-out: II. The role of position. Perception \& Psychophysics, 58, 977-991. doi:10.3758/BF03206826

Maljkovic, V., \& Nakayama, K. (2000). Priming of popout: III. A short-term implicit memory system beneficial for rapid target selection. Visual Cognition, 7, 571-595. doi:10.1080/ 135062800407202

Maylor, E. A., \& Hockey, R. (1987). Effects of repetition on the facilitatory and inhibitory components of orienting in visual space. Neuropsychologia, 25, 41-54. doi:10.1016/0028-3932 (87) $90042-X$

Mordkoff, J. T., Halterman, R., \& Chen, P. (2008). Why does the effect of short-SOA exogenous cuing on simple RT depend on the number of display locations? Psychonomic Bulletin \& Review, 15, 819-824. doi:10.3758/PBR.15.4.819

Müller, H. J., \& Rabbitt, P. M. (1989). Reflexive and voluntary orienting of visual attention: Time course of activation and resistance to interruption. Journal of Experimental Psychology: Human Perception and Performance, 15, 315-330. doi:10.1037/00961523.15.2.315

Neill, W. T., \& Valdes, L. A. (1992). Persistence of negative priming: Steady state or decay? Journal of Experimental Psychology: Learning, Memory, and Cognition, 18, 565-576. doi:10.1037/ 0278-7393.18.3.565

Posner, M. I., Snyder, C. R., \& Davidson, B. J. (1980). Attention and the detection of signals. Journal of Experimental Psychology: General, 109, 160-174. doi:10.1037/0096-3445.109.2.160

Pratt, J., \& Hommel, B. (2003). Symbolic control of visual attention: The role of working memory and attentional control settings. Journal of Experimental Psychology: Human Perception and Performance, 29, 835-845.

Qian, Q., Shinomori, K., \& Song, M. (2012). Sequence effects by nonpredictive arrow cues. Psychological Research, 76, 253-262.

Ristic, J., Friesen, C. K., \& Kingstone, A. (2002). Are eyes special? It depends on how you look at it. Psychonomic Bulletin \& Review, 9, 507-513. doi:10.3758/BF03196306

Ristic, J., \& Kingstone, A. (2012). A new form of human spatial attention: Automated symbolic orienting. Visual Cognition, 20, 244-264.

Tipper, S. P. (2001). Does negative priming reflect inhibitory mechanisms? A review and integration of conflicting views. Quarterly Journal of Experimental Psychology, 54A, 321-343. doi:10.1080/713755969

Tipples, J. (2002). Eye gaze is not unique: Automatic orienting in response to uninformative arrows. Psychonomic Bulletin \& Review, 9, 314-318. doi:10.3758/BF03196287

Wolfe, J. M., Butcher, S. J., Lee, C., \& Hyle, M. (2003). Changing your mind: On the contributions of top-down and bottom-up guidance in visual search for feature singletons. Journal of Experimental Psychology: Human Perception and Performance, 29, 483-502. doi:10.1037/0096-1523.29.2.483 